\title{
How many receptor-like kinases are required to operate a pollen tube Jorge P Muschietti ${ }^{1,2}$ and Diego L Wengier ${ }^{1}$
}

\begin{abstract}
Successful fertilization depends on active molecular dialogues that the male gametophyte can establish with the pistil and the female gametophyte. Pollen grains and stigmas must recognize each other; pollen tubes need to identify the pistil tissues they will penetrate, follow positional cues to exit the transmitting tract and finally, locate the ovules. These molecular dialogues directly affect pollen tube growth rate and orientation. Receptor-like kinases (RLKs) are natural candidates for the perception and decoding of extracellular signals and their transduction to downstream cytoplasmic interactors. Here, we update knowledge regarding how RLKs are involved in pollen tube growth, cell wall integrity and guidance. In addition, we use public data to build a pollen tube RLK interactome that might help direct experiments to elucidate the function of pollen RLKs and their associated proteins.
\end{abstract}

\begin{abstract}
Addresses
${ }^{1}$ Instituto de Investigaciones en Ingeniería Genética y Biología Molecular, Dr. Héctor Torres (INGEBI-CONICET), Vuelta de Obligado 2490, Buenos Aires C1428ADN, Argentina

${ }^{2}$ Departamento de Biodiversidad y Biología Experimental, Facultad de Ciencias Exactas y Naturales, Universidad de Buenos Aires, Int. Güiraldes 2160, Ciudad Universitaria, Pabellón II, Buenos Aires C1428EGA, Argentina
\end{abstract}

Corresponding authors: Muschietti, Jorge P (prometeo@dna.uba.ar), Wengier, Diego L (dwengier@dna.uba.ar)

Current Opinion in Plant Biology 2018, 41:73-82

This review comes from a themed issue on Growth and development Edited by Gwyneth Ingram and Ari Pekka Mähönen

For a complete overview see the Issue and the Editorial

Available online 6th October 2017

https://doi.org/10.1016/j.pbi.2017.09.008

1369-5266/@ 2017 Elsevier Ltd. All rights reserved.

\section{Introduction}

In vascular plants, most cells are bound to their neighbors from birth till death. This restriction demands a strict and complex coordination of developmental programs to create proper tissues and organs. In contrast, the male gametophyte, the pollen grain, is a simple structure that contains three cells when terminally differentiated: a vegetative cell that develops a tip-growing pollen tube to deliver the two sperm cells to the ovule. However, the apparent structural simplicity of the male gametophyte underlies a highly complex life history. Pollen tubes change neighbors throughout their lifetime and the tissues they encounter vary depending on the type of pistil on which they are competent to germinate. These can comprise dry or wet stigmas involved in pollen recognition, hydration and germination; solid or hollow styles that let pollen tubes to grow; and placental or ovule types that produce chemical signals that attract pollen tubes (Figure 1a). Each provides specific challenges that pollen tubes need to overcome in order to achieve fertilization. One of the main goals in pollen developmental biology is to uncover the mechanisms that enable pollen tube navigation in all these changing landscapes. While somatic cells use cell-to-cell communication to decide cell fate, pollen uses self-generated (autocrine) and foreign (paracrine) signals to modulate $\mathrm{Ca}^{2+}$ dynamics, actin polymerization, $\mathrm{H}^{+}$pump activity, $\mathrm{ROS}$ (reactive oxygen species) production, cell wall assembly and other processes that control pollen tube growth, guidance and sperm cell discharge [1]. In this context, signals and receptors have central roles in the life of pollen tubes.

In this review, we will focus on the putative roles of receptor-like kinases (RLKs) in mediating cell-to-cell communication during the life of pollen tubes. In our last section, we use public protein-protein interaction data to predict possible RLK functions based on their interactors. We hope the pollen development community will find our hypotheses useful as starting points for future research.

\section{RLKs in Arabidopsis pollen}

The Arabidopsis genome encodes 610 RLKs [2]. Of them, 492 are transmembrane receptor-like proteins and 118 are receptor-like cytoplasmic kinases (RLCKs). Which of these transmembrane RLKs participate in pollen signaling? Analysis of RLK gene expression throughout pollen development can provide some clues (Figure 1b). Two expression profiles stand out from the diverse patterns shown by $R L K$-encoding genes. Group 1 consists of 23 pollen-enriched RLKs whose expression increases throughout pollen development, reaching their expression maxima in mature pollen grains and/or pollen tubes. Group 2 is a small group of RLKs specifically induced after pollen tubes traverse stigmas, suggesting that these RLKs could be involved in pollen-pistil interactions. Group 1 includes some receptors with proven roles in pollen tube development, such as ANXUR1/2 (ANX1/2) [3,4], POLLEN RECEPTOR-LIKE KINASE2/3/6 (PRK2/3/6) [5 $\left.{ }^{\bullet \bullet}\right]$ and MALE DISCOVERER1/2 (MDIS1/2) [6 $6^{\bullet \bullet}$ (see below). Other RLKs in this group are RECEPTOR-LIKE 


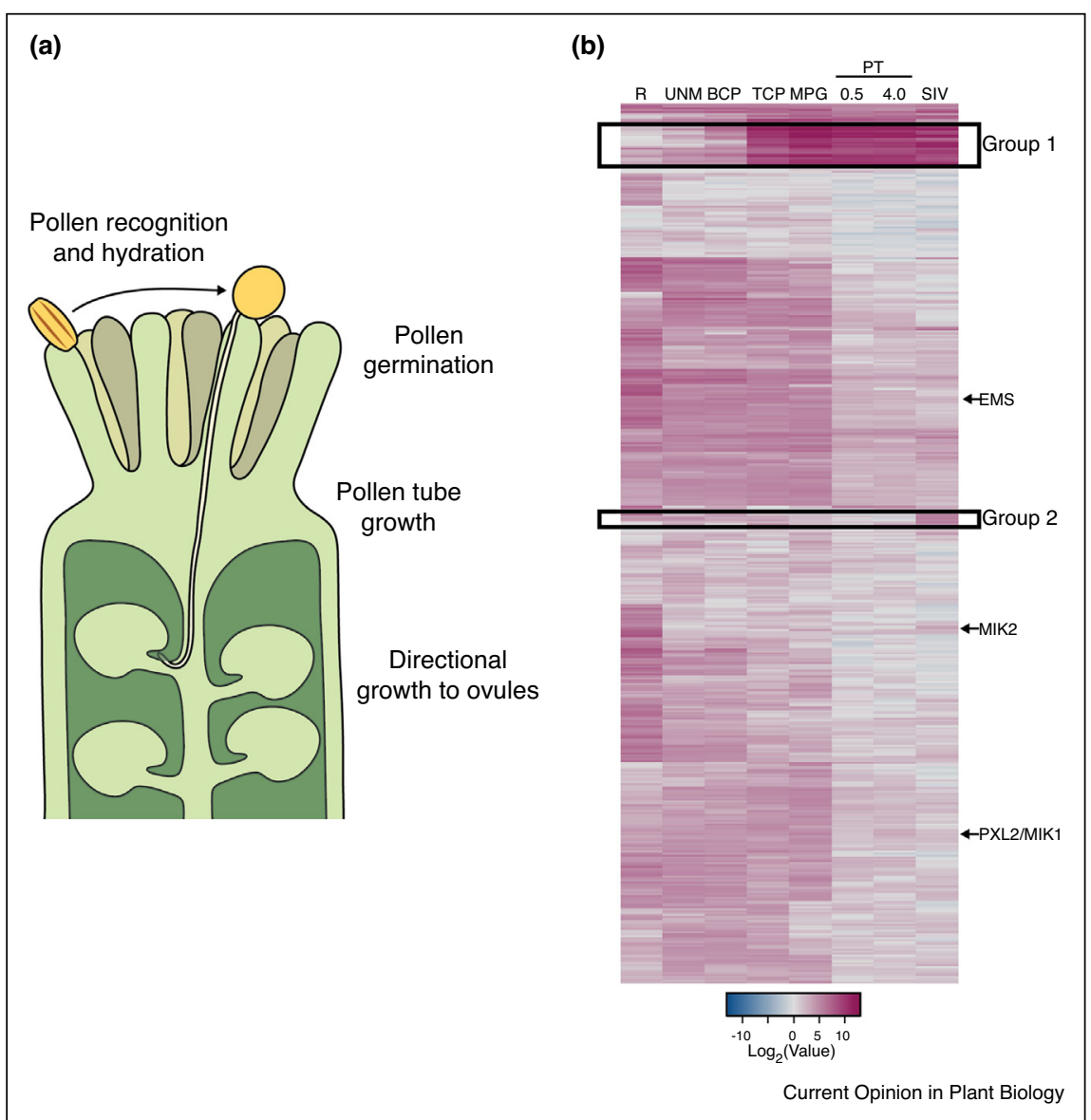

RLKs in pollen development. (a) Pollen-pistil interaction stages where pollen RLKs might be involved. (b) Expression profiles for 492 RLKencoding genes show a cluster of pollen-enriched RLK-encoding genes (Group 1) and a cluster of stigma-induced pollen RLK-encoding genes (Group 2). Expression levels in unicellular microspore (UNM), bicellular pollen (BCP), tricellular pollen (TCP), mature pollen grain (MPG) [74], two stages of in vitro-germinated pollen tubes (PT 0.5 and 4 hours) and semi in vivo-germinated pollen tubes (SIV) [42." ] were obtained from the BioAnalytic Resource (BAR, University of Toronto, Canada) and hierarchically clustered using hclust in R. Expression levels for rosette leaves (R) were included in the analysis as an indicator for baseline expression in the sporophyte. Arrows indicate other RLK-encoding genes with roles in pollen development that do not belong to Group 1 or 2.

KINASE IN FLOWERS (RKF1/2), members of the PROLINE-RICH EXTENSIN-LIKE RECEPTOR KINASES (PERKs) family [7] and some other uncharacterized RLKs. Interestingly, several RLKs that function in microgametogenesis and pollen development are not in Group 1 or 2 (indicated with arrows in Figure 1b). For example, the expression of EXCESS MICROSPOROCYTES 1 (EMS1) [8 $\left.8^{\circ}\right]$ and MDIS1-INTERACTING RECEPTOR LIKE KINASE1/2 (PXL2/MIK1 and MIK2) (see below) is not particularly enriched in the male lineage, yet mutants for these genes show abnormal male segregations. This behavior could imply that certain RLKs act as signaling modules that are recurrently recruited in various tissues (such as MIK2 $\left[9^{\circ}, 10\right]$ ).

Two fundamental questions in plant signaling hold true for pollen development: what is the meaning of having so many RLKs? What are the ligands for all these RLKs? In the next sections, we discuss the few identified ligands involved in pollen tube development and growth, and their corresponding RLKs, case by case.

\section{RLKs controlling pollen tube growth through the pistil}

Pollen-enriched RLK-encoding genes were first found in petunia (PRK1, POLLEN RECEPTOR-LIKE Kinase 1) [11] and tomato LePRK1/2 (Solanum lycopersicum, formerly Lycopersicon esculentum, POLLEN RECEPTOR-LIKE KINASE 1 and 2) [12] when searching for plasma membrane-localized kinases potentially involved in pollen development. Although similar in structure, PRK1 and LePRK1/2 have different functions. PRK1 has a role in microspore development [11] while LePRK1/2 participate in pollen tube growth and/or pollen-pistil 
interactions [13-15]. Studies of LePRK1/2 have laid the foundations to understanding certain pollen RLK signaling mechanisms. First, LePRK1/2 can form hetero-oligomeric complexes with dynamic responses to pistil components [16]. Second, several autocrine (LAT52, LATE ANTHER TOMATO 52) and paracrine (LeSTIG1, STIGMA-SPECIFIC PROTEIN 1 and STIL, STYLE INTERACTOR FOR LePRKs) ligands compete for the same RLKs [17-19]. LAT52 is a pollen cysteine-rich protein that interacts with LePRK2 only before pollen germinates [17-19]; LeSTIG1, a small style cysteine-rich protein, is a second potential ligand that can bind the extracellular domains (ECD) of LePRK1 and LePRK2, displacing LAT52 [1719]. STIL is a compound that promotes pollen tube growth presumably through LePRK2 dephosphorylation and LePRK complex disassembly [17-19]. Third, RLKs can regulate pollen tube growth rate by modulating the actin cytoskeleton through ROP-GEFs (GUANINE NUCLEOTIDE-EXCHANGE FACTORS for RHO-LIKE SMALL GTPASES OF PLANTS, ROPs) that activate ROPs [20,21]. As in tomato, Arabidopsis PRK2 interacts with and activates ROP-GEF12 [22]. In another report, PRK2 was shown to phosphorylate ROP-GEF1 in vitro as part of a PRK2/ROP-GEF1/ROP1 protein complex [23]. In contrast to the situation in tomato, however, the Arabidopsis PRK and ROP-GEF protein families seem to show higher levels of redundancy. Only prk 1 prk 2 prk 3 triple and rop-gef1 rop-gefo rop-gef12 rop-gef14 quadruple mutants produce abnormal pollen tubes compared to wildtype [23]. The current model suggests that RLKs bind to extracellular ligands and control pollen tube growth through phosphorylation-dependent activation of ROP-GEFs and ROPs, ultimately affecting actin cytoskeleton dynamics.

\section{RLKs controlling cell wall integrity during pollen tube growth}

During polarized growth, pollen tubes secrete new cell wall material to the surface of the pollen tube tip. This material is enriched in esterified pectin, resulting in a flexible cell wall that sustains tip expansion, while pectin de-esterification and callose deposition in the subapical wall provides rigidity preventing lateral expansion. In this context, a sensing mechanism for cell wall physicochemical properties is predicted to exist.

In plants, receptor-like kinases of the Catharanthus roseus RLKs (CrRLK1L) subclass have been implicated in diverse processes [24]. The most studied member of the CrRLK1L family is FERONIA (FER), an RLK expressed in all plant tissues but pollen [24,25]. ANX1 and ANX2 are two other CrRLK1Ls proposed to regulate cell wall integrity in pollen tubes [3,4]. ANX1 and $A N X 2$ are the closest homologues of $F E R$ and, together with three other yet uncharacterized CrRLK1L-encoding genes (At4g39110, At2g21480 and At5g61350), are preferentially expressed in pollen tubes [24]. Single anx1 and anx2 loss-of-function mutants do not show any visible pollen phenotype. However, anx1 anx2 double mutant is essentially sterile because pollen bursts immediately after germination [3,4]. In contrast, $A N X 1 / 2$ overexpression inhibits pollen tube growth by causing overaccumulation of cell wall material and invagination of the plasma membrane $\left[26^{\bullet \bullet}\right]$. Furthermore, ANX1-GFP and ANX2-GFP localize to the pollen tube tip membrane, a logical localization for a protein with a putative role in maintaining cell wall integrity. As for FER [27], genetic experiments place ROS production downstream of ANX1/2 signaling. In double mutants for RESPIRATORY BURST OXIDASE HOMOLOGUES H and $J$ (RBOHH and $R B O H J)$, encoding two NADPH oxidases that regulate ROS production, pollen bursts when germinated both in vitro and in vivo, like pollen of the anx1 anx2 double mutant $\left[26^{\circ \bullet}\right]$. Moreover, ANX1/2 overexpressioninduced pollen tube growth inhibition depends on $\mathrm{RBOHH} / \mathrm{J}$, suggesting a functional link between ANX1/2 and RBOHH/J [26 $6^{\circ \bullet}$. The current model is that ANX1/2 regulates ROS production in a $\mathrm{RBOHH} / \mathrm{J}-$ dependent manner, affecting calcium dynamics and subsequent deposition of new cell wall material by exocytosis $\left[26^{\circ \bullet}\right]$. This model predicts that anx1 anx2 pollen tubes should have thinner cell walls at the tips, and for this reason, burst with increasing turgor pressure. All these findings suggest that ANX1/2 act as sensors of cell wall integrity at the tip of growing pollen tubes.

A central and still unanswered question is the identity of ANX1/ANX2 ligands. The extracellular domain of the CrRLK1L family contains two malectin-like domains [28]. In animals, malectins bind di-glucose-high mannose $\mathrm{N}$-glycan (Glc2-N-glycan) [28]. If this binding capacity is functionally conserved, ANX1/2 might bind polysaccharides from the pollen tube cell wall. Contrary to this hypothesis, FER was found to bind secreted peptides of the RAPID ALKALINIZATION FACTORS family (RALF), such as RALF1 [29] and RALF-LIKE23 (RALFL23) $\left[30^{\circ \bullet}\right]$. There is a plethora of RALFLs peptides that could bind ANX1/2 and thereby play some role during pollination: RNAs encoding RALFL4/8/9/15/19/25 and 26 are preferentially expressed in pollen and RNAs encoding RALFL14/18/28 and 34 are expressed in the pistil. The presence of candidate ligands for ANX1/2 both in pollen and pistil is reminiscent of the LePRK1/2 autocrine and paracrine ligands $[17,18]$. Is there additional pollen autocrine regulation, as reported for LAT52LePRK2 [17]? Is it possible that pistil RALFLs compete with pollen RALFLs for RLK binding, as described for tomato STIG1/LAT52-LePRK2 [18], or are all these RALFLs acting simultaneously? To answer such questions, it will be imperative to determine whether pollen and/or pistil RALFLs bind ANX1/2.

Other RLKs that might be involved in sensing at the pollen cell wall are the PERKs (PROLINE-RICH 
EXTENSIN-LIKE RECEPTOR KINASES). The PERK family in Arabidopsis has 15 members and all have a proline-rich extracellular domain. PERK4/5/6/7/11 and 12 RNAs are highly expressed in pollen and, most of them are hardly detected in sporophytic tissues [7]. The link between PERKs and cell wall homeostasis was first established for PERK4, a receptor that negatively regulates root growth, sensitivity to abscisic acid (ABA) and apical dominance [31]. In membrane protein preparations from root extracts, PERK4 was released from the cell wall only after pectinase treatments. This result suggests that PERK4, and maybe all PERKs, interact with cell wall components as demonstrated for other RLKs such as WAKs (WALL-ASSOCIATED KINASE family) [32]. Future work addressing PERK functions and the nature of their ligands will reveal the potential roles of these receptors in perceiving changes in the cell wall of pollen tubes.

\section{Short-range guidance}

Plants must carry out fertilization rapidly and efficiently, minimizing the time between pollen hydration and sperm cells discharge. In their voyage to the ovary, pollen tubes need to be guided. LURE ('bait' in Japanese), cysteinerich peptides produced by synergid cells and released at the ovule micropyle, are the most extensively characterized pollen tube chemo-attractants to date [33]. In 2016, two independent research groups identified LURE1 receptors, but surprisingly, they identified different RLKs $\left[5^{\bullet \bullet}, 6^{\bullet \bullet}\right]$.

The Higashiyama group postulated that PRK6 (POLLEN RECEPTOR-LIKE KINASE6) is the bona fide LURE1 receptor because prk6 mutant pollen tubes failed to be attracted to LURE1 in vitro and because prk3 prk6 prk8 triple mutants showed abnormal fertilization in vivo $\left[5^{\circ \bullet}\right]$. They also showed that PRK6 accumulates on the pollen tube side closer to the LURE1 source before the tube changes growth direction. They demonstrated that PRK6 interacts with PRK3 and ROP-GEF12 and showed that PRK6 kinase activity is important for pollen tube growth. Moreover, PRK6 interacts with LOST IN POLLEN TUBE1 and 2 (LIP1 and LIP2, respectively), two RLCKs implicated in pollen tube growth and LURE1 signaling [34]. On the basis of the biochemical similarities to LePRK1/2, we speculate that LURE1 activates PRK6PRK3 signaling, thereby modulating ROP-GEF12 and LIP1/2 activity and affecting the actin cytoskeleton. However, it remains to be elucidated whether LURE binding to PRK6 recruits PRK3, ROP-GEF12 and LIP1/ 2 into a complex, or induces complex dissociation.

In a companion article, Wang and co-workers described two pairs of pollen RLKs, MDIS1 and MDIS2 (MALE DISCOVERER1/2), and MIK1 and MIK2 (MDIS1INTERACTING RECEPTOR LIKE KINASE1/2) as LURE1 receptors [ $6^{\circ}$ ]. They showed that $m$ dis 1 and mik1 mik2 mutant pollen were less sensitive to LURE1 guidance, although mutant pollen tube growth was otherwise normal (remarkably similar to the prk6 mutant). Interaction of LURE1 with the extracellular domains of MDIS1, MIK1 and MIK2 was demonstrated biochemically by coimmunoprecipitation and microscale thermophoresis. Moreover, LURE1 induced MDIS1-MIK1 oligomerization, activation of MIK1 kinase activity and MIK1 autophosphorylation. They also reported that LURE1 triggers down-regulation of MDIS1, by removing it from the cell membrane. In the absence of other interactors, it is difficult to predict the mechanism downstream of MDIS-MIK signaling.

It is puzzling that two overlapping complexes appear to perceive the LURE1 signal. Since prk6 and mdis mik mutants show similar abnormal guidance phenotypes, it is possible that both complexes belong to a higher order signaling unit or that they act sequentially in a complex signaling cascade. Alternatively, both complexes could regulate different aspects of pollen tube guidance. Future work elucidating downstream effectors of PRK6/PRK3 and MDIS-MIK complexes will contribute to revealing the intricacies of tip growth orientation in plant cells.

\section{Pollen phosphoproteome}

Signaling pathways frequently rely on protein phosphorylation to regulate protein-protein interactions, function, degradation and subcellular localization. Typically, ligand binding induces RLK auto-phosphorylation or trans-phosphorylation and signaling pathway activation. A phosphoproteomic analysis of Arabidopsis mature pollen identified 962 phosphorylated peptides that correspond to 598 phosphoproteins [ $\left[5^{\circ}\right]$. This list included only three pollen RLKs: two CrRLK1L pollen-specific RLKs, At2g21480 and At4g39110, and PERK6. Considering that 45 RLKs are specifically enriched in mature pollen, in addition to the presence of many other nonenriched RLKs, it is curious that only three phosphorylated receptors were identified. RLK expression levels, phospho-isoform abundance or ligand-dependent phosphorylation might explain differences between expectation and observation. In any case, future analysis of RLK phosphorylation dynamics will certainly have the potential to contribute to a better understanding of signal transduction in pollen. As shown for LePRK2 [15] and the RLKs FLAGELLIN-SENSING2/BRASSINOSTEROID INSENSITIVE1 (FLS2/BRI1) [15,36], a phosphosite mutant analysis can be used to demonstrate whether phosphorylatable residues control pollen RLK functions.

\section{Finding downstream connections for pollen RLKs}

Liu and collaborators reported that the expression of 76 RLK-encoding genes is significantly enriched in pollen tubes [34] but that only 19 of these genes affected 
male transmissions when knocked out or overexpressed. Even for these, however, the signaling mechanisms that lie downstream are mostly unknown. A common strategy for implicating proteins of unknown function in any process, is to identify their interactors, some of which may have a known activity. We took advantage of available high-throughput protein-protein interaction data [37-39] to make such predictions. Most interactions were identified from yeast two-hybrid experiments and showed different degrees of reproducibility [40,41]. For this reason, only those interactions obtained in at least three out of four replicates were included. To restrict the analysis to putative biologically relevant interactions, only interactors with relative gene expression intensities $>50$ in semi in vivo-germinated pollen tubes were included [ $42^{\circ} \circ$. Interactors for which no microarray probes are available, were also included. We also restricted the search to RLKs with a canonical structure composed of extracellular, transmembrane and kinase domains (45 out of the 76 RLKs). Only 25 of these RLKs had previously reported interactions. Finally, proteins with promiscuous interactors were eliminated. For example, At5g59650 and WALL ASSOCIATED KINASE3 (WAK3) were eliminated because they promiscuously interact with 183 and 223 proteins, respectively. A total of 125 interactions between 23 RLKs and 77 interactors remained (Figure 2). Below we highlight selected hubs and interactors whose mutants show abnormal fertility, and interactions that suggest roles for RLK activities in pollen. Information about other interactors is provided as Supplemental Information.

\section{Hubs}

- $\underline{F}$ RRIC REDUCTASE DEFECTIVE3 (FRD3) appears as a hyper-connected node in the network, interacting with ANX2, CRK26, CRK32, PRK2, PRK4, PRK5, PERK4, PERK5, At1g62950, At3g47110 and At4g29450. FRD3 is a citrate efflux transporter involved in iron nutrition. Interestingly, microspore development aborts in frd 3 mutants, but can be recovered by iron supplementation [43]. Pollen tube growth, however, was not evaluated at different iron concentrations. When not bound to citrate, free Fe participates in ROS regulation (reviewed in $[44,45]$ ). Thus, RLKs could act at three levels: by affecting iron metabolism, by regulating extracellular ROS production through regulation of citrate efflux or by controlling extracellular $\mathrm{pH}$, since frd 3 mutant shows an increase in apoplastic $\mathrm{H}^{+}$concentration [46].

\section{- $\underline{P E C T I C}$ ARABINOGALACTAN SYNTHESIS-} $\underline{R} E L A T E D(\overline{P A} G R)$ encodes an O-fucosyltransferase involved in pectin synthesis. PAGR/pagr heterozygotes do not segregate homozygotes due to a defect in pollen germination $\left[47^{\circ}\right]$. PAGR localizes to the Golgi apparatus $\left[47^{\circ}\right]$ and to vesicles like EXPOs (Exocyst-Positive Organelles) [48]. These vesicles localize in distinct puncta in the plasma membrane that are thought to release their contents to the apoplast and they contain arabinogalactan glycosyltransferases [49]. In pollen tubes, the EXPO vesicle components, SEC3 ( UNIT OF EXOCYST COMPLEX3), SEC8 (ㅁUBUNIT OF EXOCYST COMPLEX8) and EXO70A1 (EXOCYST SUBUNIT EXO70 FAMILY PROTEIN A1) localize to the tip and their mutants have defective pollen germination and tube growth $\left[47^{\bullet}, 50,51\right]$.

\section{RLK interactors}

- PRK5 interacts with GRIM REAPER (GRI) [52]. GRI is highly expressed in flowers and is processed to generate a secreted 11 amino acid peptide [52], which triggers cell death in a superoxide-dependent manner upon binding PRK5 [53]. Knockout gri plants showed reduced seed set [52], consistent with GRI-PRK5 having a role in pollen tube development. Interestingly, PRK5 also interacts with FRD3, establishing a possible downstream link to ROS production.

- GYSTEINE-RICH RLK32 (CRK32) interacts with THIOREDOXIN h5 (TRX h5) and GLUTAREDOXIN GYTOPLASMIC1 (GRXC1), two proteins from related families and with overlapping functions. TRX h5 is a cytoplasmic thioredoxin that provides resistance to $\mathrm{H}_{2} \mathrm{O}_{2}$ and regulates protein activity by reducing disulfide bridges in target proteins (reviewed in [54]). In the sporophyte $T R X h 5$ expression is increased in response to oxidative stress conditions [55] and TRX h5 is highly expressed in pollen tubes. GRXC1 is a glutathione-dependent, cytoplasmic glutaredoxin that also catalyzes disulfide oxido-reduction. The double mutant grxc1 grcx 2 has partially empty siliques, indicating that fertilization might be affected [56]. TRXs and GRXs are connected by the systems that control their redox states. Glutathione was shown to indirectly maintain the pool of reduced TRXs in

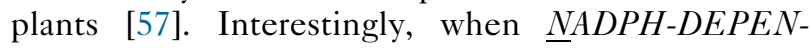
DENT TRX REDUCTASES A and B (NTRA and NTRB) are knocked out, wildtype pollen outcompetes ntra ntrb mutant pollen tubes, even though double mutants are still fertile [57]. GLUTATHIONE REDUCTASE1 (GR1) encodes one of the two GRs in Arabidopsis. It is notable that a triple mutant ntra ntrb grl fails to transmit through pollen [58]. The roles of TRXs and GRXs in pollen tubes might be controlling redox potential, protecting from ROS-induced damage or regulating the activity of interacting proteins. Alternatively, TRXs and GRXs could be acting as scaffolds for signaling, regardless of their enzymatic activity, as shown in the resistance mechanism to Cladosporium fulvum in tomato $[59,60]$.

- Two interactors of the MDIS1/2-MIK1/2 signaling complex might connect this module to the MITOGEN-ACTIVATED PROTEIN KINASE (MAPK or MPK) cascade. MIK1 interacts with At1g79570, one of seven MKKKs (MAP KINASE KINASE KINASE) in group B4 [61], although no pollen phenotype has been 
Figure 2

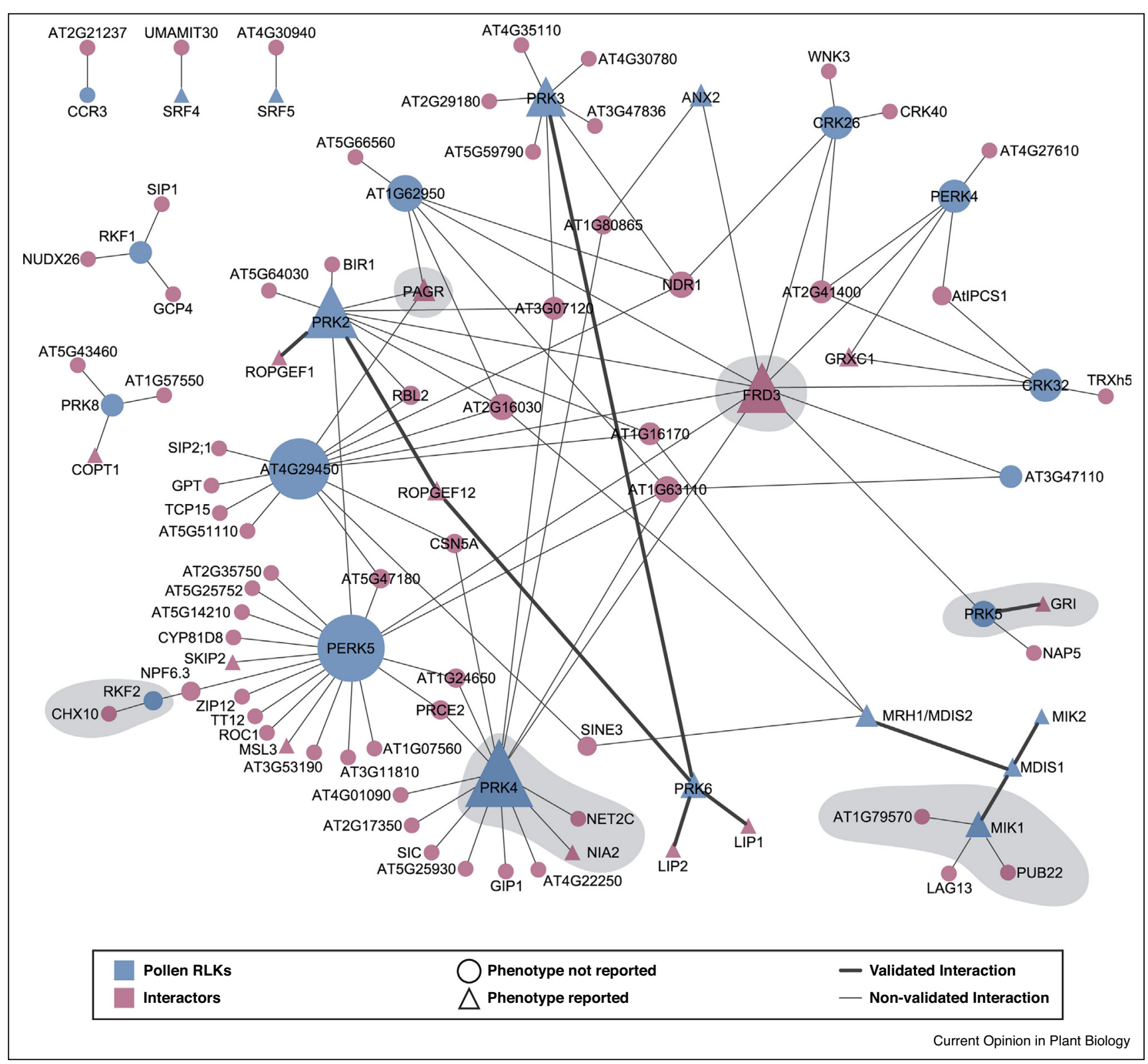

Predicted RLK pollen tube interactome. After filtering, 677 pairwise interactions remained, including those of the promiscuous RLKs, WAK3 and At5g59650, which accounted for 183 and 223 of these interactions respectively. Twenty-seven interactors were reported to interact with a large number of proteins. For example, UBQ3, At3g12180 and IQD6 interacted with 1347, 534 and 478 proteins, respectively. We eliminated proteins that had more than 100 interactors; At5g59650 and WAK3 were also eliminated. The network was generated using Cytoscape. Nodes and interactions of special interest are highlighted in grey and described in the text. Nodes sizes are proportional to their connectivity. Validated interactions are reported in the literature as pairwise interactions and often supported by genetic experiments. Non-validated interactions were mostly obtained from yeast two-hybrid experiments and in some cases, are supported by transient interaction assays in planta.

reported for mutants of this group. However, there is some support for a function of downstream MPKs in pollen tube guidance. Guan $e t$ al., showed that funicular, but not micropylar, guidance in $m p k 3 m p k 6$ mutants is defective $\left[62^{\circ}\right]$. These results argue against a role of MPK3 and MPK6 in LURE1 signaling, but do not rule out that MPK8/9/17 and 19, which show higher expression levels than $M P K 3 / 6$ in pollen tubes, could be involved. Furthermore, MKK2/3/4/5 might connect MKKKs and MPKs. MIK1 also interacts with PLANT U-BOX 22 (PUB22), establishing a second connection to MAPK signaling. PUB22, a cytoplasmic U-box E3 ubiquitin ligase, is a regulator of the immune response. PUB22 oligomerizes and auto-ubiquitinates in the 
absence of pathogens [63], but MPK3 phosphorylates and stabilizes PUB22 upon pathogen attack. Cytoplasmic accumulation of PUB22 leads to ubiquitinmediated target degradation, with targets including the EXPO component EXO70B2 [64].

- PRK4 interacts with NETWORKED2G (NET2G) and NITRATE REDUCTASE2 (NIA2). NET2G is highly expressed in pollen tubes and shares homology with the actin-binding protein NET2A [65]. NET2A is involved in actin-plasma membrane interactions and is distributed as foci at the plasma membrane of the pollen tube shank. The N-terminus of NET2 family proteins is similar to petunia KINASE-INTERACTING PROTEIN 1 (KIP1) [66], a PRK1 interactor. NIA2, together with NIA1 and NOA1 (NITRIC OXIDE ASSOCIATED1), participate in nitric oxide (NO) synthesis; fertility is affected in the nia1 nia2 noa1 triple mutant [67].

- RKF2 interacts with $\underline{\mathrm{C} A T I O N} / \underline{\mathrm{H}}^{+}$EXCHANGER10 (CHX10), a cation/ $\mathrm{H}^{+}$exchanger. CHX10 is highly expressed in pollen tubes that is predicted to be localized in the plasma membrane/vacuoles. CHX10 is related to CHX21 and CHX23, two $\mathrm{K}^{+} / \mathrm{H}^{+}$transporters also expressed in pollen tubes and with roles in guidance to ovules $[68,69]$.

\section{Conclusions and perspectives}

RLKs are important for regulating proper pollen tube growth and responding to guidance cues. The great diversity of putative RLK ligands involved in pollen tube biology, both autocrine and paracrine, suggest that competitive binding might provide positional cues that inform pollen tubes that their growth rate or path needs to be adjusted. These signals could affect $\mathrm{Ca}^{2+}$ flux, actin polymerization, $\mathrm{H}^{+}$pump activity, $\mathrm{ROS}$ production, cell wall integrity and other processes, all of which need to be synchronized and balanced to sustain tip growth and guidance. For example, exocytosis secretes cell wall precursors and other material to the plasma membrane tip, but at the same time, endocytosis maintains the vesicle stock.

Most pollen-expressed RLKs, however, have no associated function, mainly because they belong to families with high redundancy. New technologies for knocking out multiple genes at once [70], together with the use of pollen-specific promoters might provide the genetic tools for analyzing the function of complex RLK families. Moreover, to date, most RLKs are orphan receptors. What are the bona fide ligands for these RLKs? The Arabidopsis genome encodes for more than 1000 putative secreted peptides [71]. The challenge to find ligand-receptor pairs is immense. It will be necessary to design alternative, scalable techniques for high-throughput screens (e.g. [72]).
Guidance can depend on mechanisms of localized stimulation (such as PRK6-mediated growth) or inhibition (such as NO effects on growth [73]). But with $>45$ RLKs expressed in pollen tubes and a myriad of candidate ligands, how is all the information integrated in the cytoplasm? Is there a hierarchy of RLKs, with some receptors transducing more important information than others? Combinatorial mutants for several types of RLKs seem to support the idea that not all receptors are equal. For example, among PRK genes, mutations of PRK6 alone were sufficient to disrupt LURE1-mediated in vitro pollen tube guidance, while prk3 prk6 prk8 pollen tubes were less efficient than wildtype in leaving the transmitting tract (12 hours postpollination) $\left[^{\circ *}\right]$. But how can such hierarchies be established when different RLKs converge on the same downstream processes? Besides prk6 mutants, mdis 1 mdis 2 pollen tubes are also insensitive to LURE1. Considering these observations, it is evident that we are just beginning to understand the mechanisms of how RLKs regulate pollen tube development.

\section{Appendix A. Supplementary data}

Supplementary material related to this article can be found, in the online version, at http://dx.doi.org/10. 1016/j.pbi.2017.09.008.

\section{Acknowledgements}

We thank Sheila McCormick and the members of the Muschietti lab, in particular Agustina Mazzella, Laura Barberini and Ana Sede, for the critical reading of the article. This work was supported by the Agencia Nacional de Promoción Científica y Tecnológica (ANPGyT) [PICT2014-0423 and PICT2015-0078] to JPM and [PICT2015-2605] to DLW; Universidad de Buenos Aires [UBACYT-2014] to JPM.

\section{References and recommended reading}

Papers of particular interest, published within the period of review, have been highlighted as:

- of special interest

•• of outstanding interest

1. Cheung $A Y$, Wu HM: Structural and signaling networks for the polar cell growth machinery in pollen tubes. Annu Rev Plant Biol 2008, 59:547-572

2. Shiu $\mathrm{SH}$, Bleecker $\mathrm{AB}$ : Plant receptor-like kinase gene family: diversity, function, and signaling. Sci STKE 2001, 2001:re22.

3. Boisson-Dernier A, Roy S, Kritsas K, Grobei MA, Jaciubek M, Schroeder JI, Grossniklaus U: Disruption of the pollenexpressed FERONIA homologs ANXUR1 and ANXUR2 triggers pollen tube discharge. Development 2009, 136:3279-3288.

4. Miyazaki S, Murata T, Sakurai-Ozato N, Kubo M, Demura T, Fukuda H, Hasebe M: ANXUR1 and 2, sister genes to FERONIA/ SIRENE, are male factors for coordinated fertilization. Curr Biol 2009, 19:1327-1331.

5. Takeuchi H, Higashiyama T: Tip-localized receptors control

- pollen tube growth and LURE sensing in Arabidopsis. Nature 2016, 531:245-248.

This study together with $\left[6^{\circ \bullet}\right]$ demonstrates that the LURE1 peptide binds to redundant pollen tube RLKs involved in ovule targeting.

6. Wang $T$, Liang L, Xue $Y$, Jia P-F, Chen W, Zhang $M-X$, Wang $Y-C$,

-• Li H-J, Yang W-C: A receptor heteromer mediates the male perception of female attractants in plants. Nature 2016, 531:241-244. 
See annotation to Ref. [ $\left.5^{\circ \bullet}\right]$.

7. Borassi C, Sede AR, Mecchia MA, Salgado Salter JD, Marzol E, Muschietti JP, Estevez JM: An update on cell surface proteins containing extensin-motifs. J Exp Bot 2016, 67:477-487.

8. Huang J, Zhang T, Linstroth L, Tillman Z, Otegui MS, Owen HA,

- Zhao D: Control of anther cell differentiation by the small protein ligand TPD1 and its receptor EMS1 in Arabidopsis. PLoS Genet 2016, 12:e1006147.

This study shows that the small secreted cysteine-rich protein ligand (TPD1) and the RLK (EMS1) pair, control tapetal cell differentiation.

9. Van der Does D, Boutrot F, Engelsdorf T, Rhodes J, McKenna JF,

- Vernhettes S, Koevoets I, Tintor N, Veerabagu M, Miedes E et al.: The Arabidopsis leucine-rich repeat receptor kinase MIK2/ LRR-KISS connects cell wall integrity sensing, root growth and response to abiotic and biotic stresses. PLoS Genet 2017, 13:e1006832.

In this report, the receptor-like kinase MIK2 senses cell wall integrity regulating plant growth and adaptation to abiotic and biotic stresses.

10. Julkowska MM, Klei K, Fokkens L, Haring MA, Schranz ME, Testerink C: Natural variation in rosette size under salt stress conditions corresponds to developmental differences between Arabidopsis accessions and allelic variation in the LRR-KISS gene. J Exp Bot 2016, 67:2127-2138.

11. Lee H-S, Karunanandaa B, McCubbin A, Gilroy S, Kao T-h: PRK1, a receptor-like kinase of Petunia inflata, is essential for postmeiotic development of pollen. Plant J 1996, 9:613-624.

12. Muschietti J, Eyal $\mathrm{Y}, \mathrm{McC}$ Cormick S: Pollen tube localization implies a role in pollen-pistil interactions for the tomato receptor-like protein kinases LePRK1 and LePRK2. Plant Cell 1998, 10:319-330.

13. Gui CP, Dong X, Liu HK, Huang WJ, Zhang D, Wang SJ, Barberini ML, Gao XY, Muschietti J, McCormick S et al.: Overexpression of the tomato pollen receptor kinase LePRK1 rewires pollen tube growth to a blebbing mode. Plant Cell 2014, 26:3538-3555.

14. Zhang D, Wengier D, Shuai B, Gui CP, Muschietti J, McCormick S, Tang WH: The pollen receptor kinase LePRK2 mediates growth-promoting signals and positively regulates pollen germination and tube growth. Plant Physiol 2008, 148:13681379.

15. Salem T, Mazzella A, Barberini ML, Wengier D, Motillo V, Parisi G, Muschietti J: Mutations in two putative phosphorylation motifs in the tomato pollen receptor kinase LePRK2 show antagonistic effects on pollen tube length. $J$ Biol Chem 2011 286:4882-4891.

16. Wengier D, Valsecchi I, Cabanas ML, Tang WH, McCormick S, Muschietti J: The receptor kinases LePRK1 and LePRK2 associate in pollen and when expressed in yeast, but dissociate in the presence of style extract. Proc Natl Acad SciU $S$ A 2003, 100:6860-6865.

17. Tang W, Ezcurra I, Muschietti J, McCormick S: A cysteine-rich extracellular protein, LAT52, interacts with the extracellular domain of the pollen receptor kinase LePRK2. Plant Cell 2002, $14: 2277-2287$

18. Tang W, Kelley D, Ezcurra I, Cotter R, McCormick S: LeSTIG1, an extracellular binding partner for the pollen receptor kinases LePRK1 and LePRK2, promotes pollen tube growth in vitro. Plant J 2004, 39:343-353.

19. Wengier DL, Mazzella MA, Salem TM, McCormick S, Muschietti JP: STIL, a peculiar molecule from styles, specifically dephosphorylates the pollen receptor kinase LePRK2 and stimulates pollen tube growth in vitro. BMC Plant Biol 2010, 10:33.

20. Zhang D, Wengier D, Shuai B, Gui C-P, Muschietti J, McCormick S, Tang W-H: The pollen receptor kinase LePRK2 mediates growth-promoting signals and positively regulates pollen germination and tube growth. Plant Physiol 2008, 148:13681379.

21. Kaothien P, Ok SH, Shuai B, Wengier D, Cotter R, Kelley D, Kiriakopolos S, Muschietti J, McCormick S: Kinase partner protein interacts with the LePRK1 and LePRK2 receptor kinases and plays a role in polarized pollen tube growth. Plant $J$ 2005, 42:492-503.

22. Zhang $\mathrm{Y}$, McCormick $\mathrm{S}$ : A distinct mechanism regulating a pollen-specific guanine nucleotide exchange factor for the small GTPase Rop in Arabidopsis thaliana. Proc Natl Acad SciU S A 2007, 104:18830-18835.

23. Chang F, Gu Y, Ma H, Yang Z: AtPRK2 promotes ROP1 activation via RopGEFs in the control of polarized pollen tube growth. Mol Plant 2013, 6:1187-1201.

24. Boisson-Dernier A, Kessler SA, Grossniklaus U: The walls have ears: the role of plant CrRLK1Ls in sensing and transducing extracellular signals. J Exp Bot 2011, 62:1581-1591.

25. Huck N, Moore JM, Federer M, Grossniklaus U: The Arabidopsis mutant feronia disrupts the female gametophytic control of pollen tube reception. Development 2003, 130:2149-2159.

26. Boisson-Dernier A, Lituiev DS, Nestorova A, Franck CM,

-• Thirugnanarajah S, Grossniklaus U: ANXUR receptor-like kinases coordinate cell wall integrity with growth at the pollen tube tip via NADPH oxidases. PLOS Biol 2013, 11: e1001719.

This paper shows that overexpression of ANXR1/2 inhibits pollen tube growth because exocytosis is overstimulated and cell wall material at the tip area is accumulated. It also shows that ANXRs function upstream of NADPH oxidases.

27. Duan Q, Kita D, Johnson EA, Aggarwal M, Gates L, Wu HM, Cheung AY: Reactive oxygen species mediate pollen tube rupture to release sperm for fertilization in Arabidopsis. Nat Commun 2014, 5:3129.

28. Schallus $T$, Jaeckh C, Feher K, Palma AS, Liu Y, Simpson JC, Mackeen M, Stier G, Gibson TJ, Feizi T et al.: Malectin: a nove carbohydrate-binding protein of the endoplasmic reticulum and a candidate player in the early steps of protein $\mathrm{N}$ glycosylation. Mol Biol Cell 2008, 19:3404-3414.

29. Haruta M, Sabat G, Stecker K, Minkoff BB, Sussman MR: A peptide hormone and its receptor protein kinase regulate plant cell expansion. Science 2014, 343:408-411.

30. Stegmann M, Monaghan J, Smakowska-Luzan E, Rovenich $\mathrm{H}$,

-• Lehner A, Holton N, Belkhadir Y, Zipfel C: The receptor kinase FER is a RALF-regulated scaffold controlling plant immune signaling. Science 2017, 355:287-289.

This paper reports that the RALFL peptide, RALFL23 restrains immune response through the inhibitory association with FER who promotes the formation of the active FLS2/EFR-BAK1 complex.

31. Bai L, Zhang G, Zhou Y, Zhang Z, Wang W, Du Y, Wu Z, Song C-p: Plasma membrane-associated proline-rich extensin-like receptor kinase 4, a novel regulator of $\mathrm{Ca}^{2+}$ signalling, is required for abscisic acid responses in Arabidopsis thaliana. Plant J 2009, 1:314-327.

32. Kohorn BD: Cell wall-associated kinases and pectin perception. J Exp Bot 2016, 67:489-494.

33. Kanaoka MM, Higashiyama T: Peptide signaling in pollen tube guidance. Curr Opin Plant Biol 2015, 28:127-136.

34. Liu J, Zhong S, Guo X, Hao L, Wei X, Huang Q, Hou Y, Shi J, Wang C, Gu H et al.: Membrane-bound RLCKs LIP1 and LIP2 are essential male factors controlling male-female attraction in Arabidopsis. Curr Biol 2013, 23:993-998.

35. Mayank P, Grossman J, Wuest S, Boisson-Dernier A, Roschitzki B, - Nanni P, Nuhse T, Grossniklaus U: Characterization of the phosphoproteome of mature Arabidopsis pollen. Plant J 2012 72:89-101.

This was the first phosphoproteomic study of mature Arabidopsis pollen grains. In this study 598 pollen phosphoproteins were identified.

36. Jiang J, Zhang $\mathrm{C}$, Wang $\mathrm{X}$ : Ligand perception, activation, and early signaling of plant steroid receptor brassinosteroid insensitive 1. J Integr Plant Biol 2013, 55:1198-1211.

37. Chatr-aryamontri A, Breitkreutz BJ, Heinicke S, Boucher L, Winter A, Stark C, Nixon J, Ramage L, Kolas N, O’Donnell L et al.: The BioGRID interaction database: 2013 update. Nucleic Acids Res 2013, 41:D816-D823. 
38. Geisler-Lee J, O'Toole N, Ammar R, Provart NJ, Millar aH, Geisler M: A predicted interactome for Arabidopsis. Plant Physiol 2007, 145:317-329.

39. Hermjakob H, Montecchi-Palazzi L, Lewington C, Mudali S, Kerrien S, Orchard S, Vingron M, Roechert B, Roepstorff P, Valencia A et al.: IntAct: an open source molecular interaction database. Nucleic Acids Res 2004, 32:D452-D455.

40. Lalonde S, Sero A, Pratelli R, Pilot G, Chen J, Sardi MI, Parsa SA, Kim DY, Acharya BR, Stein EV et al.: A membrane protein/ signaling protein interaction network for Arabidopsis version AMPv2. Front Physiol 2010, 1:24.

41. Jones AM, Xuan Y, Xu M, Wang RS, Ho CH, Lalonde S, You CH, Sardi MI, Parsa SA, Smith-Valle E et al.: Border control - a membrane-linked interactome of Arabidopsis. Science 2014 344:711-716.

42. Qin Y, Leydon AR, Manziello A, Pandey R, Mount D, Denic S,

-• Vasic B, Johnson MA, Palanivelu R: Penetration of the stigma and style elicits a novel transcriptome in pollen tubes, pointing to genes critical for growth in a pistil. PLoS Genet 2009, 5: e1000621.

This article describes for the first time the pollen genes expressed during pollen tube growth through stigma and style tissues.

43. Roschzttardtz H, Séguéla-Arnaud M, Briat J-F, Vert G, Curie C: The FRD3 citrate effluxer promotes iron nutrition between symplastically disconnected tissues throughout Arabidopsis development. Plant Cell 2011, 23:2725-2737.

44. Cakmak I: Possible roles of zinc in protecting plant cells from damage by reactive oxygen species. New Phytol 2000, 146:185205.

45. O'Brien JA, Daudi A, Butt VS, Paul Bolwell G: Reactive oxygen species and their role in plant defence and cell wall metabolism. Planta 2012, 236:765-779.

46. Rogers EE, Guerinot ML: FRD3, a member of the multidrug and toxin efflux family, controls iron deficiency responses in Arabidopsis. Plant Cell 2002, 14:1787-1799.

47. Stonebloom S, Ebert B, Xiong G, Pattathil S, Birdseye D, Lao J,

- Pauly M, Hahn MG, Heazlewood JL, Scheller HV: A DUF-246 family glycosyltransferase-like gene affects male fertility anc the biosynthesis of pectic arabinogalactans. BMC Plant Biol 2016, 16:90.

This report reveals that a pectic arabinogalactan synthesis-related enzyme (PAGR) is essential for pollen tube growth.

48. Wang J, Ding Y, Wang J, Hillmer S, Miao Y, Lo SW, Wang X, Robinson DG, Jiang L: EXPO, an exocyst-positive organelle distinct from multivesicular endosomes and autophagosomes, mediates cytosol to cell wall exocytosis in Arabidopsis and tobacco cells. Plant Cell 2010, 22:40094030.

49. Poulsen CP, Dilokpimol A, Mouille G, Burow M, Geshi N: Arabinogalactan glycosyltransferases target to a unique subcellular compartment that may function in unconventional secretion in plants. Traffic (Copenhagen, Denmark) 2014, 15:1219-1234.

50. Cole RA, Synek L, Zarsky V, Fowler JE: SEC8, a subunit of the putative Arabidopsis exocyst complex, facilitates pollen germination and competitive pollen tube growth. Plant Physiol 2005, 138:2005-2018

51. Hála M, Cole R, Synek L, Drdová E, Pečenková T, Nordheim A, Lamkemeyer T, Madlung J, Hochholdinger F, Fowler JE et al.: An exocyst complex functions in plant cell growth in Arabidopsis and tobacco. Plant Cell 2008, 20:1330-1345.

52. Wrzaczek M, Brosché $M$, Kollist $\mathrm{H}$, Kangasjärvi J: Arabidopsis GRI is involved in the regulation of cell death induced by extracellular ROS. Proc Natl Acad Sci U S A 2009, 106:5412 5417.

53. Wrzaczek M, Vainonen JP, Stael S, Tsiatsiani L, Help-RintaRahko H, Gauthier A, Kaufholdt D, Bollhöner B, Lamminmäki A, Staes A et al:: GRIM REAPER peptide binds to receptor kinase PRK5 to trigger cell death in Arabidopsis. EMBO J 2015, 34: 55-66.
54. Gelhaye E, Rouhier N, Navrot N, Jacquot JP: The plant thioredoxin system. Cell Mol Life Sci 2005, 62:24-35.

55. Laloi C, Mestres-Ortega D, Marco Y, Meyer Y, Reichheld J-P: The Arabidopsis cytosolic thioredoxin h5 gene induction by oxidative stress and its $\mathrm{W}$-Box-mediated response to pathogen elicitor. Plant Physiol 2004, 134:1006-1016.

56. Riondet C, Desouris JP, Montoya JG, Chartier Y, Meyer Y Reichheld J-P: A dicotyledon-specific glutaredoxin GRXC1 family with dimer-dependent redox regulation is functionally redundant with GRXC2. Plant Cell Environ 2012, 35:360-373.

57. Reichheld J-P, Khafif M, Riondet C, Droux M, Bonnard G, Meyer $Y$ : Inactivation of thioredoxin reductases reveals a complex interplay between thioredoxin and glutathione pathways in Arabidopsis development. Plant Cell 2007, 19:1851-1865.

58. Marty L, Siala W, Schwarzländer M, Fricker MD, Wirtz M, Sweetlove LJ, Meyer Y, Meyer AJ, Reichheld J-P, Hell R: The NADPH-dependent thioredoxin system constitutes a functional backup for cytosolic glutathione reductase in Arabidopsis. Proc Natl Acad Sci U S A 2009, 106:9109-9114.

59. Nekrasov V, Ludwig AA, Jones JDG: CITRX thioredoxin is a putative adaptor protein connecting Cf-9 and the ACIK1 protein kinase during the Cf-9/Avr9-induced defence response. FEBS Lett 2006, 580:4236-4241.

60. Rivas S, Rougon-Cardoso A, Smoker M, Schauser L, Yoshioka H, Jones JDG: CITRX thioredoxin interacts with the tomato Cf-9 resistance protein and negatively regulates defence. EMBO J 2004, 23:2156-2165.

61. Ichimura K, Shinozaki K, Tena G, Sheen J, Henry Y, Champion A, Kreis M, Zhang S, Hirt H, Wilson $\mathrm{C}$ et al.: Mitogen-activated protein kinase cascades in plants: a new nomenclature. Trends Plant Sci 2002, 7:301-308.

62. Guan Y, Lu J, Xu J, McClure B, Zhang S: Two mitogen-activated

- $\quad$ protein kinases, MPK3 and MPK6, are required for funicular guidance of pollen tubes in Arabidopsis. Plant Physiol 2014, 165:528-533.

Here, evidence is provided that pollen MPK3 and MPK6 are involved in mediating the guidance response during fertilization. Pollen of the double mutant mpk3 mpk6 has reduced transmission.

63. Furlan G, Nakagami H, Eschen-Lippold L, Jiang X, Majovsky $P$, Kowarschik K, Hoehenwarter W, Lee J, Trujillo M: Changes in PUB22 ubiquitination modes triggered by MITOGENACTIVATED PROTEIN KINASE3 dampen the immune response. Plant Cell 2017, 29:726-745.

64. Stegmann M, Anderson RG, Ichimura K, Pecenkova T, Reuter P, árský V, McDowell JM, Shirasu K, Trujillo M: The ubiquitin ligase PUB22 targets a subunit of the exocyst complex required for PAMP-triggered responses in Arabidopsis. Plant Cell 2012, 24:4703-4716.

65. Deeks MJ, Calcutt JR, Ingle EKS, Hawkins TJ, Chapman S, Richardson AC, Mentlak DA, Dixon MR, Cartwright F, Smertenko AP et al.: A superfamily of actin-binding proteins at the actin-membrane nexus of higher plants. Curr Biol 2012 22:1595-1600

66. Skirpan AL, McCubbin AG, Ishimizu $T$, Wang $X, H u Y$, Dowd PE, $\mathrm{Ma} \mathrm{H}$, Kao T: Isolation and characterization of kinase interacting protein 1, a pollen protein that interacts with the kinase domain of PRK1, a receptor-like kinase of petunia. Plant Physiol 2001, 126:1480-1492.

67. Lozano-Juste J, León J: Enhanced abscisic acid-mediated responses in nia1nia2noa1-2 triple mutant impaired in NIA/ NR- and AtNOA1-dependent nitric oxide biosynthesis in Arabidopsis. Plant Physiol 2010, 152:891-903.

68. Evans AR, Hall D, Pritchard J, Newbury HJ: The roles of the cation transporters $\mathrm{CHX} 21$ and $\mathrm{CHX} 23$ in the development of Arabidopsis thaliana. J Exp Bot 2012, 63:59-67.

69. Lu Y, Chanroj S, Zulkifli L, Johnson MA, Uozumi N, Cheung A, Sze H: Pollen tubes lacking a pair of $\mathbf{K}+$ transporters fail to target ovules in Arabidopsis. Plant Cell 2011, 23 . $81-93$ 
70. Zhang Z, Mao Y, Ha S, Liu W, Botella JR, Zhu JK: A multiplex CRISPR/Cas9 platform for fast and efficient editing of multiple genes in Arabidopsis. Plant Cell Rep 2016, 35:1519-1533.

71. Lease KA, Walker JC: The Arabidopsis unannotated secreted peptide database, a resource for plant peptidomics. Plant Physiol 2006, 142:831-838.

72. Butenko MA, Wildhagen M, Albert M, Jehle A, Kalbacher $\mathrm{H}$, Aalen RB, Felix G: Tools and strategies to match peptide-ligand receptor pairs. Plant Cell 2014, 26:1838-1847.

73. Prado AM, Porterfield DM, Feijo JA: Nitric oxide is involved in growth regulation and re-orientation of pollen tubes. Development 2004, 131:2707-2714.

74. Honys D, Twell D: Comparative analysis of the Arabidopsis pollen transcriptome. Plant Physiol 2003, 132:640-652. 